\title{
Dust colors, dust release rates, and dust-to-gas ratios in the comae of six comets ${ }^{\star}$
}

\author{
G.C. Sanzovo ${ }^{1}$, P.D. Singh ${ }^{2,3}$ and W.F. Huebner ${ }^{2}$ \\ ${ }^{1}$ Department of Physics, State University of Londrina, Perobal, 86051 Londrina, PR, Brazil \\ 2 Southwest Research Institute, 6220 Culebra Road, P. O. Drawer 28510, San Antonio, TX 78228-0510, U.S.A. \\ Walter.huebner@hq.nasa.gov \\ 3 On leave from Department of Astronomy, Institute of Astronomy and Geophysics, University of São Paulo, Av. \\ Miguel Stefano 4200, Agua Funda, São Paulo, Cep. 04301-904, Brazil
}

Received February 9, 1995; accepted March 22, 1996

\begin{abstract}
In an attempt to find correlations between properties of comets, we determine the dust colors, dust release rates, and dust-to-gas mass ratios in the comae of Comets Bowell $(1980 \mathrm{~b}=1982 \mathrm{I})$, Bradfield $(1979 \mathrm{l}=$ 1979 X), Brorsen-Metcalf (1989 o = 1989 X), Giacobini-Zinner (1984 e = 1985 XIII), Levy $(1990 \mathrm{c}=1990 \mathrm{XX})$, and Stephan-Oterma $(1980 \mathrm{~g}=1980 \mathrm{X})$ using a uniform and systematic analysis of the continuum fluxes measured at optical wavelengths. We assume that the continuum fluxes observed in these comets are from light scattered by micrometer-sized dust particles. The color of the dust is blue, relative to the Sun, in Comets Bradfield (1979 X) and Brorsen-Metcalf (1989 X); both comets have a low dust-to-gas mass ratio $(\chi<10 \%)$. Comets Giacobini-Zinner (1985 XIII) and Stephan-Oterma $(1980 \mathrm{X})$ have an intermediate dust-to-gas mass ratio $(10 \%<\chi<40 \%)$, whereas Comets Levy (1990 XX) and Bowell (1982 I) have a high dust-to-gas mass ratio ( $\chi>40 \%$ ). The continuum flux from dust particles in comets with intermediate to high dust-to-gas mass ratios is in general red, relative to the Sun. However, the color changes to blue in these comets when the dust-to-gas mass ratios are low relative to their peak values.
\end{abstract}

Key words: comets - Bowell (1982 I); Bradfield (1979 X); Brorsen-Metcalf (1989 X); Giacobini-Zinner (1985 XIII); Levy (1990 XX); Stephan-Oterma (1980 X)

\section{Introduction}

Spacecraft-based in situ measurements combined with ground-based observations of Comet $\mathrm{P} /$ Halley revealed one of the most important discoveries in cometary science: Dust contributes gas to the coma in addition to that released directly from the nucleus. From the measured cometocentric distributions of $\mathrm{CO}$ (Eberhardt et al. 1987), CN (A'Hearn et al. 1986a,b), $C_{2}$ (A'Hearn et al. 1986a,b), and $\mathrm{H}_{2} \mathrm{CO}$ (Snyder et al. 1989; Boice et al. 1990; Meier et al. 1993) in Comet Halley it was concluded that dust particles are direct as well as indirect sources of each of these species in the coma. Vaisberg et al. (1986) noticed a distinct reduction of the dust size distribution with distance from the nucleus in Comet Halley and proposed fragmentation of larger dust particles near the nucleus $(R<10000$ $\mathrm{km})$ with accompanying enhancement of gas release. Dust

Send offprint requests to: W.F. Huebner

*Tables 1-3 only available in electronic form can be accessed by ftp at cdsarc.u-strasbg.fr or 130.79.128.5 or on www at http://u-strasbg.fr/abstract.html characteristics may change as dust particles move away from the nucleus in the coma and also as the heliocentric distance of the comet varies during its apparition. Dust release rates and the related dust-to-gas mass ratios in the coma are important parameters for determining element abundances of a comet (Geiss 1988; Jessberger et al. 1989). In an effort to find correlations between some properties of comets, we present in this paper a systematic and uniform analysis of continuum fluxes observed at optical wavelengths in the comae of Comets Bowell (1980 b $=1982 \mathrm{I})$, Bradfield $(1979 \mathrm{l}=1979 \mathrm{X})$, Brorsen-Metcalf $(1989 o=1989$ X), Giacobini-Zinner $(1984$ e $=1985$ XIII $)$, Levy $(1990 \mathrm{c}=1990 \mathrm{XX})$, and Stephan-Oterma (1980 g $=1980 \mathrm{X})$. We determine dust release rates, characteristic dust colors, and dust-to-gas mass ratios including their variations with heliocentric distances.

\section{Theoretical considerations}

Dust release rates: It has been shown that the continuum flux at optical wavelengths observed in the spectrum 
of the coma of a comet is due to solar light scattered by dust. To determine the dust release rates from the nucleus, based on continuum measurements in the coma, we use the same method as described by de Freitas Pacheco et al. (1988), Landaberry et al. (1991), and Singh et al. (1992). It was noted by Newburn and Spinrad (1989) and also by us (Singh et al. 1996) that the comet nucleus makes a significant contribution to the continuum flux, especially when the phase angle of the comet is small. The phase function of the nucleus can be represented by

$$
\phi_{\mathrm{N}}(\theta)=\phi_{1} \cdot \mathrm{e}^{-u \cdot \theta},
$$

where $\phi_{1}=0.9982, u=1.842 \mathrm{rad}^{-1}$, and $\theta$ is the phase angle in radian. Representing the nucleus by a sphere with equivalent radius $R_{\mathrm{N}}$, the nuclear contribution to the product of area, $A_{\mathrm{N}}$, and geometric albedo is

$$
A_{\mathrm{N}} \cdot p(\lambda) \cdot \phi_{\mathrm{N}}(\theta)=\pi R_{\mathrm{N}}^{2} \cdot p(\lambda) \cdot \phi_{\mathrm{N}}(\theta),
$$

where $p(\lambda)$ is the geometric albedo of the nucleus. The phase function of the nucleus and the nuclear contribution $A_{\mathrm{N}} \cdot p(\lambda) \cdot \phi_{\mathrm{N}}(\theta)$ to the product of area and geometric albedo at the dates of observations of the six comets under discussion were calculated from Eqs. (1) and (2) and are listed in Cols. 3 and 4, respectively, of Table 2 . The observed product of area and geometric albedo of the dust coma plus nucleus were computed from Eq. (5) of Singh et al. (1992), using the measured continuum flux as listed in the Col. 2 of Table 2 at wavelength $\lambda$. The values of $A \cdot p(\lambda) \cdot \phi(\theta)$ of the dust coma plus nucleus are listed in Col. 5 of Table 2. Subtracting the nuclear contribution, and dividing by the scattering function, we list the product of area and geometric albedo $A_{\mathrm{d}} \cdot p(\lambda)$ for the dust coma alone in the last column of the Table 2 . Using these $A_{\mathrm{d}} \cdot p(\lambda)$ values, we solved Eq. (1) coupled with Eqs. (2) to (4) of Singh et al. (1992) numerically to determine the dust release rates as listed in Table 3 . In Table 1, we list the data sets, date (UT), heliocentric distance $(r)$, geocentric distance $(\Delta)$, phase angle $(\theta)$, and projected diaphragm radius $(s)$ at the coma for each observation. These values were taken from A'Hearn et al. (1981, 1984), Baratta et al. (1991), Schleicher et al. $(1987,1991)$ and Millis et al. (1982), for Comets Bowell (1980 b = $1982 \mathrm{I})$, Bradfield (1979 l = 1979 X), Brorsen-Metcalf $(1989$ o = 1989 X), GiacobiniZinner $(1984 \mathrm{e}=1985 \mathrm{XIII})$, Levy $(1990 \mathrm{c}=1990 \mathrm{XX})$, and Stephan-Oterma $(1980 \mathrm{~g}=1980 \mathrm{X})$. The parameters $M$ and $\phi(\theta)$ were calculated according to the prescriptions of de Freitas Pacheco et al. (1988) and Singh et al. (1992). In Table 3 we summarize gas release rates, maximum sizes of dust particles $\left(a_{\mathrm{m}}\right)$ that can be entrained by the coma gas, effective mean dust particle sizes $(a)$ responsible for the continuum emission at wavelength $\lambda$ (see below), dust release rates, and mass ratios of the dust-to-gas release rates $\chi$ for the data sets of Table 1 . Water is the dominant gaseous species in the coma; in situ measurements in the coma of Comet Halley have shown that about $80 \%$ of the gas is $\mathrm{H}_{2} \mathrm{O}$. Thus, we assumed for the coma composition $77 \% \mathrm{H}_{2} \mathrm{O}, 13 \% \mathrm{CO}$, and $10 \%$ other gases of mean molecular weight $30 \mathrm{amu}$ consisting mainly of $\mathrm{CO}_{2}, \mathrm{NH}_{3}$, $\mathrm{CH}_{3} \mathrm{OH}, \mathrm{N}_{2}, \mathrm{H}_{2} \mathrm{CO}, \mathrm{S}_{2}, \mathrm{CS}_{2}, \mathrm{H}_{2} \mathrm{~S}$, and HCN. For this composition the mass gas release rate $q_{\mathrm{g}}$ (in units of $\mathrm{g} / \mathrm{s}$ ) is

$$
q_{\mathrm{g}}=3.4010^{-23} Q\left(\mathrm{H}_{2} \mathrm{O}\right),
$$

where $Q\left(\mathrm{H}_{2} \mathrm{O}\right)$ is the release rate of the number of water molecules per second. To determine the dust mass release rates and mass ratios of the dust-to-gas release rates, the model requires estimates for the nuclear radius, relative size of the active nuclear surface area, albedo, and density of dust particles - parameters that are not well known (Newburn \& Spinrad 1985; Singh et al. 1992). We present here a systematic approach to determine the dust mass release rates and the mass ratios of the dust-to-gas release rates assuming an albedo $p(\lambda)=0.04$ and a mean density $\rho_{\mathrm{d}}=1 \mathrm{~g} / \mathrm{cm}^{3}$ for the dust particles. We describe estimates of other model parameters for the six comets separately.

(1) Comet Bowell (1980 b = 1982 I): Several estimates of the average nuclear diameter of Comet Bowell (1982 I) are available (A'Hearn et al. 1984; Jewitt 1984; Bailey et al. 1992). A CCD photometric image taken on November 3, 1986, at record heliocentric distance $r=13.6 \mathrm{AU}$ shows a total cross section of $710^{11} \mathrm{~cm}^{2}$ (Meech \& Jewitt 1987) for the nucleus assuming a visual geometric albedo $p=0.06$ (Veeder \& Hanner 1981). This indicates that the effective nuclear radius of the comet is $R_{\mathrm{N}} \approx 5$ $\mathrm{km}$. Optical and infrared observational constraints, for a slowly rotating water ice nucleus at perihelion (A'Hearn et al. 1984; Jewitt 1984), indicate that the nuclear radius is between 3 and $6.5 \mathrm{~km}$, if the whole sunlit side of the nucleus was active. We have adopted $R_{\mathrm{N}} \approx 4 \mathrm{~km}$ for the continuum flux analysis and considered the extreme case that the entire sunlit hemisphere of the nuclear surface is active.

To estimate the gas mass release rates from Eq. (3), we utilized Fig. 6 of A'Hearn et al. (1984) for the OH production rates at the heliocentric distances of the comet listed in Table 1 and considered that about $85 \%$ of the photodissociations of $\mathrm{H}_{2} \mathrm{O}$ lead to $\mathrm{OH}$ at solar maximum (Huebner et al. 1992). The gas release rates calculated from Eq. (3) are listed in the second column of Table 3 . The dust mass release rates analyzed from the continuum measurements of A'Hearn et al. (1984) at $\lambda=5240 \AA$, assuming a geometric albedo of 0.04 for dust particles (Singh et al. 1992; de Freitas Pacheco et al. 1988; Landaberry et al. 1991), and the dust-to-gas mass ratios in the coma, at the dates of observations listed in Table 1, are given in Cols. 5 and 6 of Table 3 , respectively.

(2) Comet Bradfield $(1979 \mathrm{l}=1979$ X): Newburn \& Spinrad (1989) analyzed their continuum flux measurements of February 7 and March 17, 1980, in the 4780-4830 $\AA$ bandpass filter considering an effective nuclear radius of $1 \mathrm{~km}, 10 \%$ active fraction of the surface area, and either 
$\rho_{\mathrm{d}}=\rho_{\mathrm{N}}=1.0 \mathrm{~g} / \mathrm{cm}^{3}$ for a geometric albedo $p=0.03$, or the product of $\rho_{\mathrm{d}}$ and $\rho_{\mathrm{N}}$ equal to $0.3 \mathrm{~g}^{2} / \mathrm{cm}^{6}$ for $p=0.04$ for the coma dust. We assumed the same nuclear radius and fraction of active surface area. The product of $\rho_{\mathrm{d}}$ and $\rho_{\mathrm{N}}$ enters the equation for the dust release from the nucleus.

We estimate the $\mathrm{OH}$ production rates for the heliocentric distance range $0.57<r<0.86 \mathrm{AU}$ from the power law fit $\left(3.4510^{28} r^{-3.2}\right)$ of A'Hearn et al. (1981). Following the analysis of Comet Bowell (1982 I), the gas production rates were determined from Eq. (3) and are listed in Table 3 for the dates of observations by A'Hearn et al. (1981). The dust release rates, calculated from continuum fluxes measured at $\lambda=5240 \AA$, and the dust-to-gas mass ratios in the coma are listed in Cols. 4 and 5 of Table 3 , respectively.

(3) Comet Brorsen-Metcalf $(1989 \mathrm{o}=1989 \mathrm{X})$ : Jewitt \& Luu (1990) detected a flux of $90 \pm 18 \mathrm{mJy}$ at $\lambda=0.8$ $\mathrm{cm}$ and analyzed the submillimeter continuum flux. They report an average nuclear radius $R_{\mathrm{N}}=10 \mathrm{~km}$ for Comet Brorsen-Metcalf (1989 X). DiSanti \& Fink (1991) measured the OI $6300 \AA$ line flux on July $13,1989,(r=1.36$ $\mathrm{AU})$ and August 2, 1989, $(r=1.01 \mathrm{AU})$ and derived $\mathrm{H}_{2} \mathrm{O}$ release rates of $2.5710^{28}$ and $7.0010^{28}$ molecules $\mathrm{s}^{-1}$, respectively. They showed that the $\mathrm{H}_{2} \mathrm{O}$ release rates of Comet Brorsen-Metcalf before perihelion were about $15 \%$ of those of $\mathrm{P} /$ Halley at about the same values of $r$ (see Table II of DiSanti \& Fink 1991) and probably obey the same $r^{-2.54}$ power law.

Since the present continuum flux analysis (see below) corresponds to the period August 4 to 19, 1989, we chose August $2,1989,(r=1.01 \mathrm{AU})$ to derive $Q\left(\mathrm{H}_{2} \mathrm{O}\right) \approx$ $7.010^{28}$ molecules $\mathrm{s}^{-1}$. At $r=1.01 \mathrm{AU}$, the water vaporization model of Delsemme (1982) yields a water flux of $3.210^{17}$ molecules $\mathrm{cm}^{-2} \mathrm{~s}^{-1}$. For a spherical nucleus with $R_{\mathrm{N}}=10 \mathrm{~km}$, the theoretical water flux and estimated water production rate indicate that about $2 \%$ of the nuclear surface area was active in early August 1989. Considering a $r^{-2.54}$ power law for the water production rate and $2 \%$ active fraction of the surface area, we determine dust release rates, gas production rates, and ratios of dust-to-gas mass release rates as shown in Table 3.

(4) Giacobini-Zinner (1984 e = $1985 \mathrm{XIII})$ : Comet Giacobini-Zinner (1985 XIII) has been studied by many investigators. The most comprehensive photometric study was made by Schleicher et al. (1987) from data obtained between June 15.4 to November 20.5, 1985. They observed continuum emissions at 3650 and $4845 \AA$ and emissions from $\mathrm{NH}, \mathrm{OH}, \mathrm{CN}, \mathrm{C}_{2}$, and $\mathrm{C}_{3}$ at optical wavelengths through different circular diaphragms at the 31 , 42 , and 72 inch telescopes at Lowell Observatory. We have analyzed their continuum fluxes measured at 3650 and $4845 \AA$, considering an average nuclear radius of $1.2 \mathrm{~km}$ (Landaberry et al. 1991), 10\% active fraction of the spheri- cal surface area (Newburn \& Spinrad 1989), and an albedo $p \approx 0.04$ for the dust.

Following the analyses of Comets Bowell (1982 I) and Bradfield (1979 X), we estimate the gas mass production rates, Eq. (3), from the water release rates derived from $\mathrm{OH}$ production rates of Schleicher et al. (1987). Table 1 lists observing and dust model parameters adopted for the comet and Table 2 lists the continuum flux observations (Schleicher et al. 1987), phase functions of the nucleus, the nucleus contribution to the continuum, the nucleus plus dust coma continuum, and the dust coma continuum alone. Table 3 lists the gas production rates, dust release rates, and ratios of dust-to-gas mass release rates derived from the analysis of the continuum flux measurements at $4845 \AA$ of Schleicher et al. (1987).

(5) Comet Levy $(1990 \mathrm{c}=1990 \mathrm{XX})$ : Ground-based narrow band filter photometry of Comet Levy (1990 XX) in the period from June 3, 1990, to April 19, 1991, has been performed by Schleicher et al. (1991) who reported continuum flux measurements through different diaphragms at wavelengths 3650 and $4845 \AA$.

We have analyzed their continuum fluxes on the basis that the active fraction of the surface is $6 \%$ for an equivalent spherical nucleus with $R_{\mathrm{N}} \approx 3 \mathrm{~km}$ (Cowan \& A'Hearn 1979; Schleicher et al. 1991). To determine the gas production rates from Eq. (3), we used the $\mathrm{OH}$ production rates of Schleicher et al. (1991) derived from a Haser model at the dates of observations. Dust release rates were determined from the analysis of the continuum flux measurements at $\lambda=4845 \AA$ and are shown in Table 3 .

(6) Comet Stephan-Oterma $(1980 \mathrm{~g}=1980 \mathrm{X})$ : Comet Stephan-Oterma $(1980 \mathrm{X})$ is a short period comet with a period of 38 years. Its last apparition occurred in 1980/81; at perihelion (December 5,1980 ) its heliocentric and geocentric distances were $r=1.574 \mathrm{AU}$ and $\Delta=0.596 \mathrm{AU}$, respectively. Narrowband filter photometry of the comet was performed at Lowell and Mauna Kea observatories by Millis et al. (1982) during the period from September, 1980, to April, 1981. They measured the continuum fluxes at wavelengths $3300,3675,4120$, and $5240 \AA$. Following Newburn \& Spinrad (1989), we analyzed their continuum fluxes assuming $10 \%$ active fraction of the surface of a spherical nucleus with $R_{\mathrm{N}} \approx 5 \mathrm{~km}$. The $\mathrm{H}_{2} \mathrm{O}$ release rates were derived from $\mathrm{OH}$ production rates of Millis et al. (1982), considering that $\mathrm{OH}$ comes from the photodissociation of $\mathrm{H}_{2} \mathrm{O}$ with a branching ratio of about $85 \%$ (Huebner et al. 1992). These $\mathrm{H}_{2} \mathrm{O}$ release rates were used in Eq. (3) to derive gas mass release rates at the listed dates of observations and to derive dust release rates that are listed in Table 3 for the continuum flux analysis at $\lambda=5240 \AA$.

Dust colors: If $m_{c}\left(\lambda_{1}\right)$ and $m_{c}\left(\lambda_{2}\right)$ are observed magnitudes of a comet at continuum wavelengths $\lambda_{1}$ and $\lambda_{2}$, then for $\lambda_{1}<\lambda_{2}$ the observed color index, $C I$, of the 
comet is

$$
C I=m_{\mathrm{c}}\left(\lambda_{1}\right)-m_{\mathrm{c}}\left(\lambda_{2}\right),
$$

and the color excess $C E$ of comet dust relative to the Sun is

$$
C E=C I-\left[m_{\odot}\left(\lambda_{1}\right)-m_{\odot}\left(\lambda_{2}\right)\right],
$$

where the term in brackets in Eq. (5) is the solar color index at the same wavelengths as in the comet. In terms of observed continuum fluxes, the color excess of dust can be written as

$$
C E=-2.5 \log X
$$

where

$$
X=\frac{F_{\mathrm{c}}\left(\lambda_{1}\right) F_{\odot}\left(\lambda_{2}\right)}{F_{\odot}\left(\lambda_{1}\right) F_{\mathrm{c}}\left(\lambda_{2}\right)} .
$$

In terms of the quantity Af $\rho$ (A'Hearn et al. 1984), $X$ in Eq. (6) is

$$
X=\frac{[A f \rho]_{\lambda_{1}}}{[A f \rho]_{\lambda_{2}}} .
$$

In Eq. (7), $F_{\odot}\left(\lambda_{1}\right)$ and $F_{\odot}\left(\lambda_{2}\right)$ are the solar fluxes at wavelengths $\lambda_{1}$ and $\lambda_{2}$, and $F_{\mathrm{c}}\left(\lambda_{1}\right)$ and $F_{\mathrm{c}}\left(\lambda_{2}\right)$ are the corresponding continuum fluxes from the comet. Thus for $\lambda_{1}<\lambda_{2}$, the color of the dust coma of a comet is red, solar type (neutral), or blue relative to the Sun if $C E$ is positive, about zero, or negative, respectively.

Equivalent dust particle sizes: If all dust particles in the coma had the same size, the equivalent particle radius can be obtained from the dust mass loss rate (Table 3 ) and the product of the observed area and geometric albedo of particles (Table 2) at a given heliocentric distance, $r$. This single parameter provides a convenient criterion for comparing different comets. If the expansion velocity of the dust in the coma is represented by the empirical relation $v_{\mathrm{d}} \approx 0.5 r^{-0.6} \mathrm{~km} / \mathrm{s}$ (Bobrovnikoff 1954; Jewitt et al. 1982) then the dust will travel a distance $s$ in $\mathrm{km}$ in a time

$$
t=2 s \cdot r^{0.6},[\mathrm{~s}] \text {. }
$$

The equivalent particle radius, $a$, responsible for the dust mass release rate is obtained from

$$
q_{\mathrm{d}}=\frac{4 \pi a^{3} \rho_{\mathrm{d}} N}{3 t},
$$

where $\rho_{\mathrm{d}}$ is the density of the equivalent dust particle and $N$ is the number of dust particles contained in the cross sectional area, $A_{\mathrm{d}}$, covered by the diaphragm at the coma of the comet. From the observed product of area and geometric albedo $A_{\mathrm{d}} \cdot p(\lambda)$ (Table 2), we have

$$
N=\frac{A_{\mathrm{d}} \cdot p(\lambda)}{0.04 \pi a^{2}}
$$

where we have assumed that $p(\lambda)=0.04$ for the dust (Keller et al. 1986). Solving Eqs. (9) to (11) gives the equivalent particle radius. The density, $\rho_{\mathrm{d}}$, of a particle is not known. We assume $\rho_{\mathrm{d}}=1 \mathrm{~g} / \mathrm{cm}^{3}$.

$$
a=\frac{0.06 q_{\mathrm{d}} \cdot s \cdot r^{0.6}}{\rho_{\mathrm{d}} \cdot A_{\mathrm{d}} \cdot p(\lambda)} .
$$

\section{Results and discussions}

(1) Comet Bowell $(1980 \mathrm{~b}=1982 \mathrm{I})$ : Comet Bowell (1982 I) was discovered by E. L. G. Bowell on February $11.32292,1980$, through a $0.33 \mathrm{~m}$ photographic telescope when the comet was near Jupiter and was +16.5 magnitude bright (IAU circ. 3461). The comet was "new" in the Oort sense $(1 / a)_{\text {orig }}=+0.00004 \mathrm{AU}^{-1}$ and had its perihelion at $q=3.188509 \mathrm{AU}$ on April 3.8954, 1982. Comet Bowell differs from other comets; it was dust-rich and exhibited substantial activity at $r>6$ AU. Numerous hypotheses have been put forward to explain the existence of a coma at large $r$ (Meech \& Jewitt 1987; Huebner et al. 1993; Mukai 1986; Yabushita \& Wada 1987). None of these give clear explanations for the high activity of Comet Bowell at such large heliocentric distances.

Comet Bowell (1982 I) was observed through optical filters by A'Hearn et al. (1984) during the period November, 1980, to June, 1982. They measured the continuum fluxes of the coma of the comet through different circular diaphragms at wavelengths 3300,3675 , and $5240 \AA$. We have re-analyzed their continuum fluxes measured at $5240 \AA$ on the basis that the nucleus is a sphere with $R_{N}$ $\approx 4 \mathrm{~km}$ and its whole sunlit side was active during the period November, 1980, to June, 1982, when the phase angle of the comet varied from $0.2^{\circ}$ to $17.2^{\circ}$.

As found by A'Hearn et al. (1984), the color excess of the dust in the coma is red relative to the Sun in the wavelength range $3675-5240 \AA$ for the period November $14.52,1980$, to June $24.32,1982$. The dust is also redder than the Sun in the larger wavelength range $3300-$ $5240 \AA$ except on March 12.36, 1981, (through a 19.6 arcsec diaphragm) and on April 30.45, 1982, (through a 9.6 arcsec diaphragm). On these two dates the color excess of the dust is blue relative to the Sun. On April 30.45, 1982 , observations through a 9.6 arcsec diaphragm show a color excess $C E=+0.87^{\mathrm{mag}}$ (red relative to the Sun) and $-1.15^{\mathrm{mag}}$ (blue relative to the Sun), for wavelength ranges $3300-3675 \AA$ and $3675-5240 \AA$, respectively, but the color excess is bluer relative to the Sun for the combined wavelength range $3300-5240 \AA$. A similar effect was noticed in the continuum flux measurements of A'Hearn et al. (1984) on March 12.36, 1981, (through a 19.6 arcsec diaphragm) $C E=-0.37^{\mathrm{mag}}$ (blue relative to the Sun) and $C E=+0.34^{\mathrm{mag}}$ (red relative to the Sun) for wavelength ranges $3300-3675 \AA$ and $3675-5240 \AA$, respectively. The change of color may be related to a change in size distribution or composition of dust from one wavelength range to the other wavelength range. The effective particle sizes for the dust release rates, consistent with the observed product of geometrical area and albedo, indicate $a=9.1$ and $5.5 \mu \mathrm{m}$ on March 12.36, 1981, and April 30.45, 1982 , respectively.

The high activity of Comet Bowell (1982 I) during the pre-perihelion heliocentric distance range 5.254 to 4.630 $\mathrm{AU}$ is also noticed in high dust release rates (Table 3 ). In 
Fig. 1 we present the variation of dust release rate with heliocentric distance for Comet Bowell (1982 I). On April 6.39, 1981, $(r=4.630 \mathrm{AU})$ Comet Bowell was losing dust at a rate of $3.24410^{6} \mathrm{~g} / \mathrm{s}$. A similarly high gas production rate was obtained by A'Hearn et al. (1984) with an $\mathrm{OH}$ production rate of $1.5510^{29}$ molecules $\mathrm{s}^{-1}$ on April 5 to 6 , 1981. The temporal increase in dust and gas production rates on April 5 to 6, 1982, may be caused by a "sporadic burst" that occurred in the comet. For the period April 6,1981 , to January 30,1982 , no continuum flux data are available for the comet. Hence, the dust and gas activity of the comet for this 9-month period is unknown. Comet Bowell (1982 I) showed again its high activity in dust release rates April 28 to 30, 1982, when diaphragms used in observations by A'Hearn et al. (1984) covered projected distances $s>9500 \mathrm{~km}$ from the nucleus at the comet (Tables 3 and 1). Since $\chi>40 \%$, the comet belongs to the family of comets with high dust-to-gas ratios.

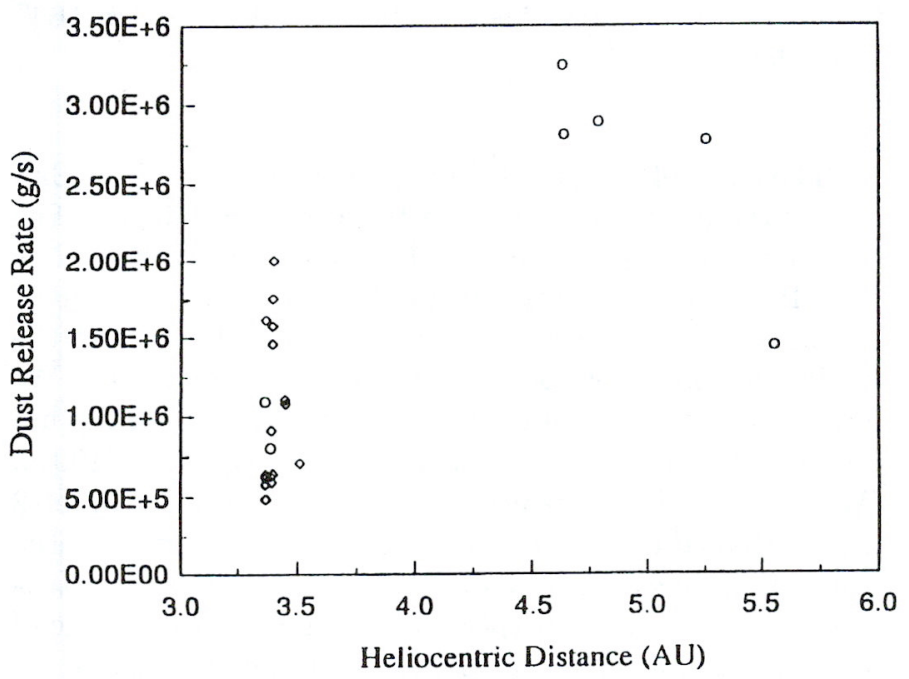

Fig. 1. Variation of dust release rate with heliocentric distance for Comet Bowell (1982 I). Circles: Pre-perihelion, diamonds: Post-perihelion

(2) Comet Bradfield (1979 l = 1979 X): Comet Bradfield (1979 X) was discovered on December 24, 1979, in South Australia (IAU Circ. 3437). It has an elliptical orbit with semi-major axis of order of $43 \mathrm{AU}$ and a period of about 280 years. The perihelion of the comet occurred on December 21.6 UT, 1979, at $q=0.5453$ AU. The comet was closest to the Earth $(\Delta=0.198$ AU) on January 26,1980 . Post-perihelion narrow band filter photometry of the comet was carried out from Perth, Mauna Kea, and Flagstaff observatories by A'Hearn et al. (1981). OH, CN, $\mathrm{C}_{3}$, and $\mathrm{C}_{2}$ production rates were determined from emission band fluxes measured at optical wavelengths on the basis of the Haser model formalism. Production rates of each of these molecular species obey a $r^{-3.2 \pm 0.5}$ power law (A'Hearn et al. 1981). A'Hearn et al. (1981) have also mea- sured continuum fluxes at wavelengths $3300,3675,3930$, 4120 , and $5240 \AA$ through different diaphragms and have reported equivalent widths of the $\Delta v=0$ sequence of the $\mathrm{C}_{2}$ Swan bands which they claim are a measure of the upper limit of the gas-to-dust ratios in the coma of the comet. The dust release rates for Comet Bradfield (1979 X) were not estimated by them.

Post-perihelion dust release rates, estimated as described above, vary from $3.3410^{4} \mathrm{~g} / \mathrm{s}$ (January 18.83, 1980) to $4.4110^{5} \mathrm{~g} / \mathrm{s}$ (December 29.82, 1979) in the coma of Comet Bradfield (1979 X). In Fig. 2 we present the variation of dust release rate, $q_{\mathrm{d}}$, with heliocentric distance, $r$, for Comet Bradfield (1979 X). The December 29.82, 1979, observations probably indicate a small burst of activity on a time scale of one day. It appears that dust release rates follow two different power laws, with a change in the exponent at $r \approx 0.9 \mathrm{AU}$ in late January, 1980. A similar conclusion was drawn for the $\mathrm{C}_{2}$ production rates by A'Hearn et al. (1981) and visual magnitude measurements of the comet as discussed by Morris (1980). We find $q_{\mathrm{d}}$ to vary as $r^{-5.3 \pm 0.4}$ and $r^{-1.2 \pm 0.2}$ for $0.57<r<0.86$ $\mathrm{AU}$ and $0.86<r<1.666 \mathrm{AU}$, respectively. On January 18.66, 1980, $(r=0.816 \mathrm{AU})$ and January 20.92, 1980, $(r=0.848 \mathrm{AU})$, the comet showed brightnesses $5.8^{\mathrm{mag}}$ and $4.8^{\mathrm{mag}}$, respectively. The increase in brightness could be related to a burst event that occurred on January 19 - 20, 1980. Our calculations indicate low dust-to-gas ratios, $\chi$, on February $1.12,1980$, and also for the period January 16.80 to $18.83,1980$, which is in agreement with $\mathrm{C}_{2}$ equivalent width estimates of A'Hearn et al. (1981). On January $20.81,1980, q_{\mathrm{d}}$ as well as $\chi$ increased by a factor of 2 relative to their corresponding values of January 18.83 , 1980. A similar effect has also been noticed by A'Hearn et al. (1981), which they explain as probably due to contamination of $5240 \AA$ continuum flux by $\mathrm{NH}_{2}$ emission of $0,12,0$ and $1,8,0$ bands. To check this possibility, we calculated dust release rates using the continuum flux at $4120 \AA$ of A'Hearn et al. (1981) where there are no strong $\mathrm{NH}_{2}$ bands. We find no evidence for an increase of $q_{\mathrm{d}}$ or $\chi$ on January $20.81,1980$, relative to January 17.82 to $18.83,1980$. Since $\chi<10 \%$ Comet Bradfield (1979 X) belongs to the family of comets with low mass ratios of dust-to-gas release rates.

For the observation on February 7, 1980, $(r=1.14 \mathrm{AU}$, $\Delta=0.51 \mathrm{AU})$, Newburn and Spinrad (1989) analyzed continuum fluxes measured in the $4780-4830 \AA$ bandpass and found a dust release rate of $23.56 \mathrm{~kg} / \mathrm{s}$ and $\chi=0.069$ for model parameters: $\rho_{\mathrm{d}} \rho_{\mathrm{N}}=0.3 \mathrm{~g}^{2} / \mathrm{cm}^{6}, p=0.04$, and $M=15$. For the observation on February 6.25, 1980, we find a dust release rate of about $72 \mathrm{~kg} / \mathrm{s}$ and $\chi=0.08$ by analyzing the continuum flux at $\lambda=5240 \AA$ of A'Hearn et al. (1981), using parameters $\rho_{\mathrm{d}} \rho_{\mathrm{N}}=0.5 \mathrm{~g}^{2} / \mathrm{cm}^{6}, p=0.04$, and $M=15$. In view of the different $\rho_{\mathrm{d}} \rho_{\mathrm{N}}$ and continuum bandpass, we consider our $q_{\mathrm{d}}$ and $\chi$ values consistent with those of Newburn \& Spinrad (1989). Estimates of 


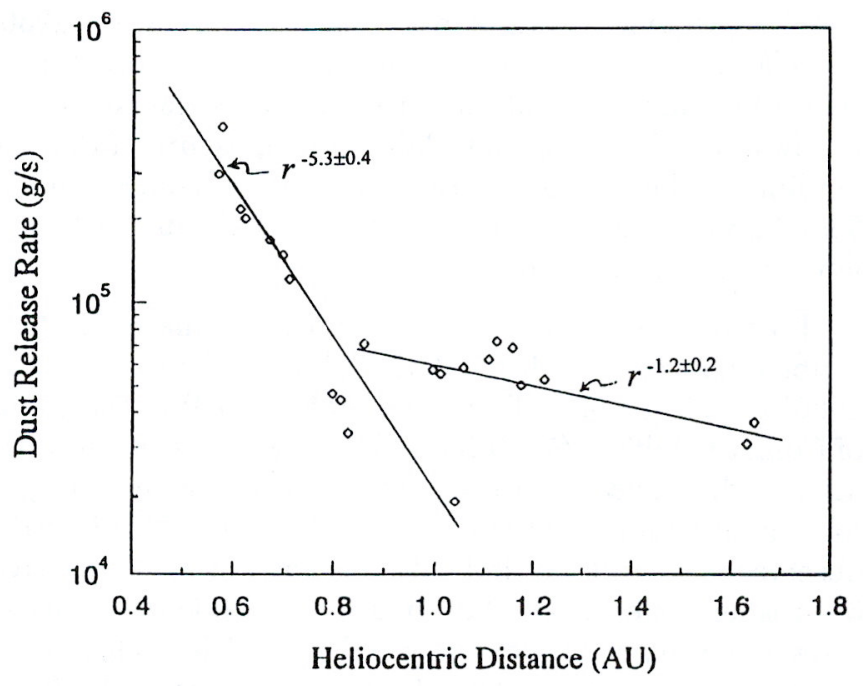

Fig. 2. Variation of dust release rate with heliocentric distance post-perihelion for Comet Bradfield (1979 X)

equivalent sizes of dust particles for both the low dust release rate (February 1.12, 1980) and the high dust release rate (December 29.82, 1979) indicate $a \approx 4 \mu \mathrm{m}$ and a blue color excess (Eq. 6) of the dust relative to the Sun for all observing wavelengths of A'Hearn et al. (1981) for the period December 28.83, 1979, to March 10.13, 1980.

(3) Comet Brorsen-Metcalf $(1989 \mathrm{o}=1989 \mathrm{X})$ : Although the period, perihelion distance, and eccentricity of Comet Brorsen-Metcalf is similar to that of $\mathrm{P} / \mathrm{Halley}$, a study by DiSanti \& Fink (1991), comparing the compositions, shows that they differ in physical and chemical characteristics. Pre-perihelion photometric observations of Comet Brorsen-Metcalf were performed through circular diaphragms of 41.4 and 138 arcsec at the 91-cm Cassegrain telescope of the Catania Astrophysical Observatory by Baratta et al. (1991) from August 4 to 19, 1989. They determined relative dust release rates in arbitrary units by the method of A'Hearn \& Cowan (1975) using the continuum flux measurements at $\lambda=4845 \AA$.

They find an increase in dust release rates with decreasing $r$ in the range $0.72<r<0.97$ (AU). The dust mass release rate obeys a $r^{-2.4 \pm 0.2}$ power law. Our dust release rates, determined from their continuum flux measurements at $\lambda=4845 \AA$ through 41.4 and 138 arcsec diaphragms, are shown in Table 3. In Fig. 3 we present the variation of dust release rate with heliocentric distance for Comet Brorsen-Metcalf (1989 X). The dust release rates can be represented by a fit

$$
q_{\mathrm{d}}=1.6910^{5} \cdot r^{-2.0 \pm 0.5},[\mathrm{~g} / \mathrm{s}] .
$$

It is evident that the dust release rates increase with decreasing $r$ in Comet Brorsen-Metcalf (1989 X). The comet showed maximum and minimum dust release rates on $\mathrm{Au}-$ gust 19 and August 4, 1989, respectively.

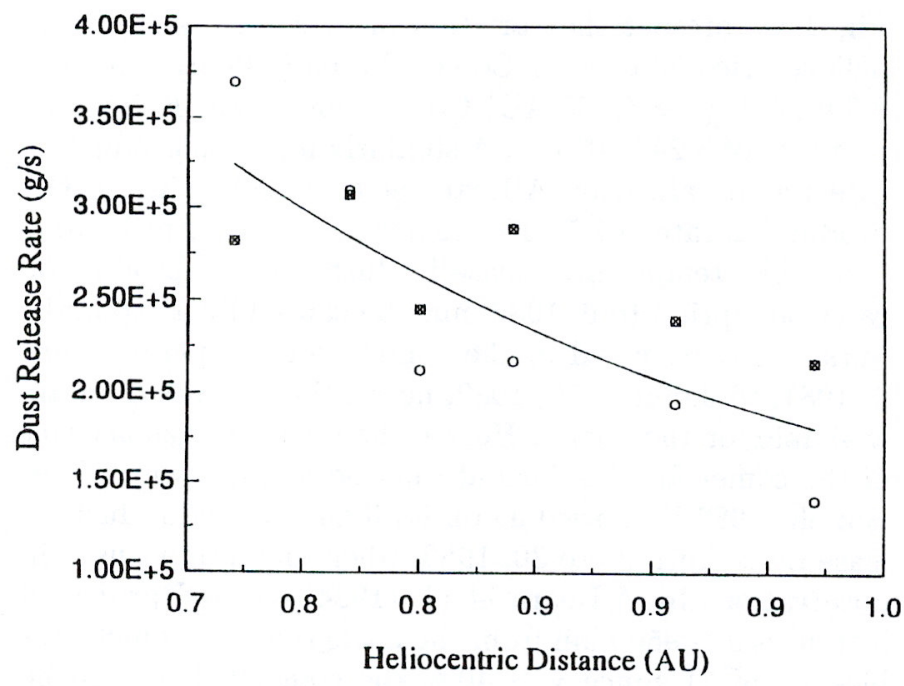

Fig. 3. Variation of dust release rate with heliocentric distance for Comet Brorsen-Metcalf (1989 X). Open circles: 41.4" diaphragm, Crossed circles: $138^{\prime \prime}$ diaphragm

The color of the dust is blue relative to that of the Sun during the observing period of Baratta et al. (1991). The dust mass release rates in Comet Brorsen-Metcalf are less than $10 \%$ of the gas mass production rates. Since $\chi<10 \%$ (Table 3) Comet Brorsen-Metcalf (1989 X) belongs to the family of comets with low dust-to-gas mass ratios. We have also estimated dust release rates using continuum flux measurements at $\lambda=3650 \AA$ of Baratta et al. (1991). We find that these values of $q_{\mathrm{d}}$ are up to a factor of 2 higher than the corresponding values estimated from the continuum flux at $\lambda=4845 \AA$. With the increase of $q_{\mathrm{d}}$ is a related increase in $A_{\mathrm{d}} \cdot p(\lambda)$, hence the equivalent size of dust particles as given by Eq. (12) remains unaltered.

An estimate of dust particle size in the coma of Comet Brorsen-Metcalf $(1989 \mathrm{X})$ shows that emissions at $\lambda=$ 4845 and $3650 \AA$ are due to dust particles with $a \approx 3.5$ $\mu \mathrm{m}$. We did not find any evidence of outburst activity or short term variation at a level larger than $25 \%$ in dust release rates during the pre-perihelion period August 4 to 19,1989 . Our continuum flux analysis at $4845 \AA$ shows that the dust release rates were about $110^{5}$ to $310^{5} \mathrm{~g} / \mathrm{s}$ in the coma of Comet Brorsen-Metcalf (see Table 3). In addition, continuum emissions at $4845 \AA$ and at 3675 $\AA$ were due to light scattered by abundant small grains with $a \approx 3.5 \mu \mathrm{m}$ in the coma of the comet. Our estimated radii of grains and dust release rates in August 1989 are consistent with the lower limit of particle radius 1 to $2 \mu \mathrm{m}$ and dust production rate $q_{\mathrm{d}} \approx 310^{5} \mathrm{~g} / \mathrm{s}$ recently set by IR studies of Comet Brorsen-Metcalf (1989 X) near perihelion by Lynch et al. (1992). A comparison of dust release rates of Comet Brorsen-Metcalf (this paper) with that of Comet Halley (Singh et al. 1992) shows that dust release rates in Comet Brorsen-Metcalf are about two 
orders of magnitude smaller than those of Comet Halley. This is consistent with the conclusion drawn by Gehrz \& Ney (1992) from IR studies of P/Halley (1986 III) and six recent bright comets including $\mathrm{P} /$ Brorsen-Metcalf.

(4) Comet Giacobini-Zinner (1984 e = $1985 \mathrm{XIII})$ : In Fig. 4 we present the variation of dust release rate with heliocentric distance for Comet Giacobini-Zinner (1985 XIII). For the pre-perihelion period June 15.4 to September 9.481, 1985, the dust release rate increases with decreasing heliocentric distance, reaches a maximum of $q_{\mathrm{d}}=4.6910^{5} \mathrm{~g} / \mathrm{s}$ on August 11.434, 1985, at $r=1.087$ $\mathrm{AU}$ and then decreases with decreasing $r$ until September $09.481,1985$, to $q_{\mathrm{d}}=1.5810^{5} \mathrm{~g} / \mathrm{s}$. The dust release rate increases by a factor of about 3 early in August, 1985, relative to that on September 9, 1985. A similar increase by a factor of 2.3 is also evident in the $\mathrm{OH}$ production rate (see Table 4 of Schleicher et al. 1987), the CN production rate (Schleicher et al. 1987), and the $J_{0}$ magnitude (see Fig. 7 of Hanner et al. 1992) at about 30 days before perihelion passage of the comet. It appears that the comet suffered an outburst in early August, 1985, as it moved inbound to perihelion. Our calculations (Table 3) show that the comet was losing dust at a rate of about $1.510^{5} \mathrm{~g} / \mathrm{s}$ on September 12, 1985, and had a dust-to-gas mass ratio of $\chi \approx 0.2$. A recent study of $1-20 \mu \mathrm{m}$ photometry of the comet by Hanner et al. (1992) shows corresponding values of $110^{5} \mathrm{~g} / \mathrm{s}$ and $\chi \approx 0.11$ for September 12.6, 1985. In view of the different methods adopted by Hanner et al. (1992), we consider the agreement good.

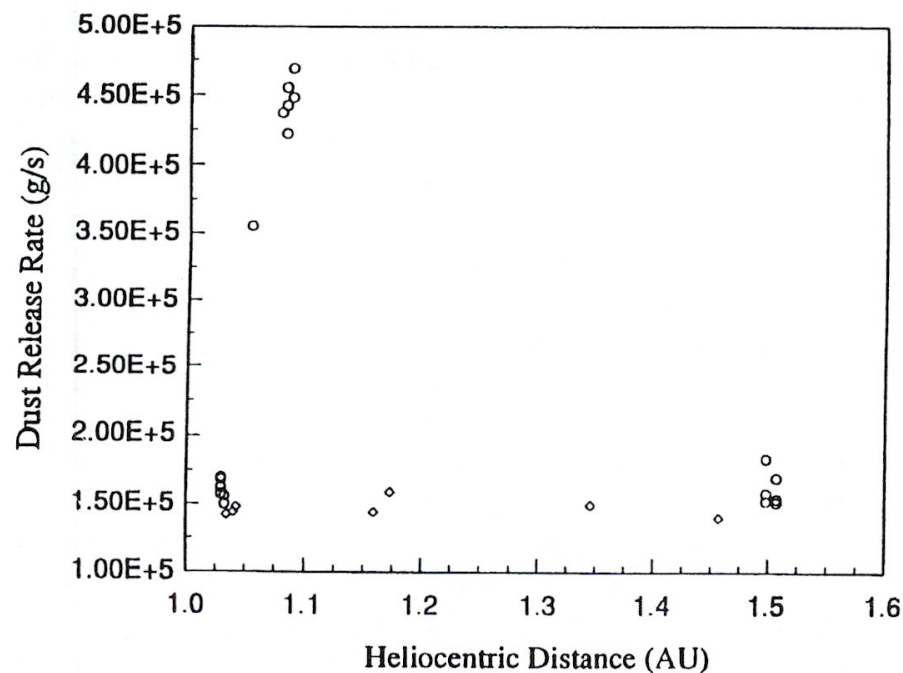

Fig. 4. Variation of dust release rate with heliocentric distance for Comet Giacobini-Zinner (1985 XIII). Circles: Pre-perihelion, diamonds: Post-perihelion

The continuum emissions at $\lambda=4845 \AA$ before perihelion were due to dust particles of equivalent radius $a \approx 4.5$ $\mu \mathrm{m}$ and for the post-perihelion period (Schleicher et al. 1987; McFadden et al. 1987) the continuum was due to dust particles of effective radius $a \approx 3.5 \mu \mathrm{m}$. A similar conclusion was drawn by Hanner et al. (1992) who reported that the infrared emission was due to micron-sized absorbing grains for a dust release rate $q_{\mathrm{d}} \approx 10^{5} \mathrm{~g} / \mathrm{s}$. The dust release rates of the comet during the observing period June to November, 1985, of Schleicher et al. (1987) was about $30 \%$ of the gas mass release rates (see Table 3 ). Since $\chi$ lies between $10 \%$ and $40 \%$, Comet GiacobiniZinner (1985 XIII) belongs to the family of comets with intermediate dust-to-gas mass ratios. In general, the color of the dust is red relative to that of the Sun. For the September 13.457, 1985, observation of Schleicher et al. (1987) we find $C E$ of the dust blue by $0.2^{\mathrm{mag}}$ relative to the Sun. The color of the dust was redder relative to the Sun in JHK photometry (Hanner et al. 1992).

(5) Comet Levy $(1990 \mathrm{c}=1990 \mathrm{XX})$ : Comet Levy (1990 XX) was discovered on May 20.44, 1990, by D.H. Levy with the $0.4-\mathrm{m}$ reflector in Tucson, AZ, and was $9.6^{\mathrm{m}}$ bright at the time of its discovery. The perihelion of the comet occurred on October 24.6954 ET, 1990, at $q=0.938581$ AU (Marsden 1990). Current determina-

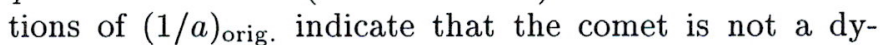
namically new comet in the Oort sense (Marsden 1990). However; pre- to post-perihelion asymmetry characteristics similar to those of other long-period comets (Whipple 1978; Roettger et al. 1989) and a high pre-perihelion $\mathrm{OH}$ production rate long before that of other species, suggest that the 1990/1991 apparition of Comet Levy (1990 XX) may be the first in the inner solar system (Schleicher et al. 1991).

Inspection of the dust release rates of Comet Levy (1990 XX) during the period June 3, 1990, to April 19, 1991 , shows that the comet was very dusty compared to other comets (see Table 3). Comet Levy's dust release rates decrease with increasing $r$ and can be expressed by the relationship

$$
q_{\mathrm{d}}=q_{0} r^{-n},
$$

where $q_{0}=8.9410^{6} \mathrm{~g} / \mathrm{s}$ and $n=2.2 \pm 0.3$ for postperihelion. The $q_{0}$ and $n$ values for pre-perihelion are $1.0110^{7} \mathrm{~g} / \mathrm{s}$ and $n=0.9 \pm 0.1$, respectively. We find an asymmetry in $q_{\mathrm{d}}$ about perihelion in Comet Levy. At $r \approx 1 \mathrm{AU}$, dust release rates are $8.9410^{6} \mathrm{~g} / \mathrm{s}$ and $1.0110^{7}$ $\mathrm{g} / \mathrm{s}$ for post- and pre-perihelion, respectively. The dust release rate $q_{\mathrm{d}}$ is higher pre-perihelion compared to postperihelion at a given $r$ (see for example, dust release rates in Table 3 for June 3.41, 1990, and March 17.53, 1991; August 21.62, 1990 and December 27.76, 1990; and August 22.57, 1990, and December 26.75, 1990). Clearly, Comet Levy (1990 XX) had a more active dust release pre-perihelion than post-perihelion. A similar effect has also been noted in the determination of $\mathrm{OH}, \mathrm{NH}, \mathrm{CN}, \mathrm{C}_{3}$, and $\mathrm{C}_{2}$ production rates by Schleicher et al. (1991). In Figs. 5a and 5b we present the variation of the dust release rate with heliocentric distance of Comet Levy (1990 XX) for pre-perihelion and post-perihelion $r$, respectively. It is 
evident from Table 3 that a mean value of $q_{\mathrm{d}} \approx 7.510^{6}$ $\mathrm{g} / \mathrm{s}$ is found for Comet Levy (1990 XX) for the period August 18.27 to $30.57,1990$. This is in agreement with values $9.310^{6}$ and $8.710^{6} \mathrm{~g} / \mathrm{s}$ derived recently by Lynch et al. (1992) for the dust release rates on 12 August and 14 August, 1990, respectively, from $8-\mu \mathrm{m}$ to $13-\mu \mathrm{m}$ spectrophotometry of Comet Levy (1990 XX). Since $\chi>40 \%$, Comet Levy (1990 XX) belongs to the family of comets with high dust-to-gas ratios.
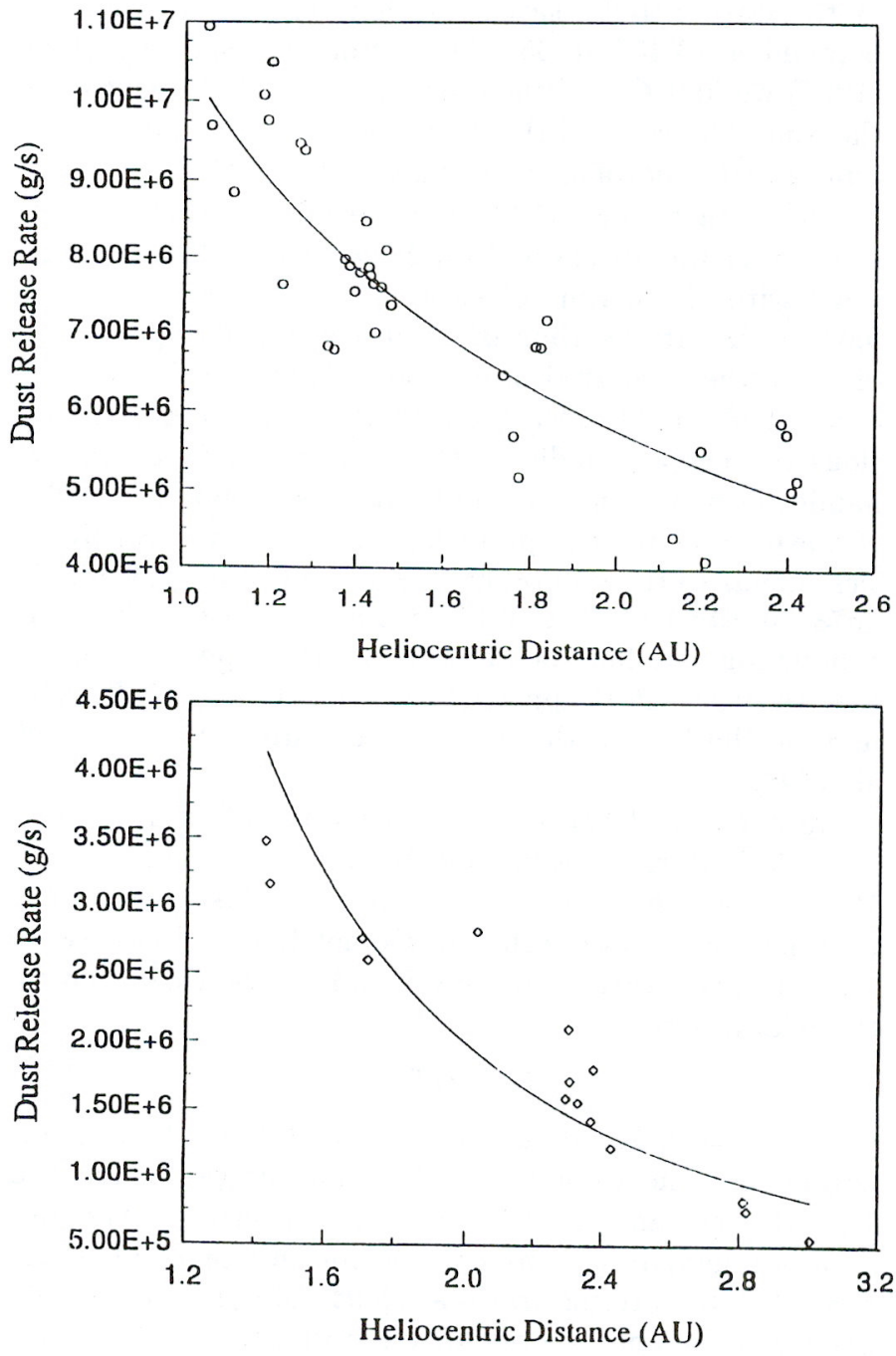

Fig. 5. Variation of dust release rate with heliocentric distance for Comet Levy (1990 XX). a) Pre-perihelion, b) post-perihelion

A calculation of the effective size of dust particles responsible for the continua at $\lambda=3650$ and $4845 \AA$ gives a range $a=6$ to $12 \mu \mathrm{m}$. The particle radii decrease with decreasing $r$ inbound to perihelion and then increase with increasing $r$ outbound. The particles were bigger in general inbound than outbound. For September 26 to 27, 1990, we estimate an average particle size $a \approx 5.5 \mu \mathrm{m}$, which is con- sistent with the dust particle size $3.3 \mu \mathrm{m}$ estimated from Eq. (3) of Fulle et al. (1992) for their listed parameters. In addition, our particle size estimate compares well with $a=5.9$ to $20 \mu \mathrm{m}$ assuming a grain density $\rho_{\mathrm{d}}=1 \mathrm{~g} / \mathrm{cm}^{3}$ obtained by Weaver et al. (1992) from imaging the inner coma of Comet Levy (1990 XX) with the Hubble Space Telescope.

The color of the dust remained blue relative to the Sun for a period of about two months before and about 3 months after perihelion. Comet Levy (1990 XX) showed variability in dust color relative to the Sun both before and after perihelion.

(6) Comet Stephan-Oterma $(1980 \mathrm{~g}=1980 \mathrm{X})$ : Inbound to perihelion the dust release rates increase with decreasing $r$. Near perihelion, Comet Stephan-Oterma was losing dust at a rate of about $250 \mathrm{~kg} / \mathrm{s}$. In Fig. 6a we present the variation of dust release rate with heliocentric distance for this comet pre- and post-perihelion.

The dust release rate can be fitted by

$$
q_{\mathrm{d}}=7.7610^{7} r^{-12.4 \pm 1.4} .
$$

In Fig. $6 \mathrm{~b}$ we present the variation of dust release rate with heliocentric distance for Comet Stephan-Oterma (1980 X) for the pre-perihelion heliocentric distance range $1.575<$ $r<1.950 \mathrm{AU}$ only. This dust release rate can be fitted by

$$
q_{\mathrm{d}}=5.0810^{6} r^{-6.8 \pm 0.7} .
$$

A similar power law $\left(r^{-5.3 \pm 1}\right)$ was determined by Millis et al. (1982). It is interesting to note the activity of Comet Stephan-Oterma (1980 X) near perihelion by comparing the dust release rates determined on November 14.41 and November 8.35, 1980. During this period of about one week, the dust release rate increased by $40 \%$. Minimum and maximum dust release rates were determined at $r=2.170 \mathrm{AU}$ (April 6.22, 1981) and at $r=1.575$ AU (December 1.29, 1980), respectively. The dust mass release rates varied between 20 to $30 \%$ of the gas mass release rates in Comet Stephan-Oterma (see Table 3) for the whole observing period of Millis et al. (1982). Since $\chi$ lies between $10 \%$ and $40 \%$, Comet Stephan-Oterma (1980 X) belongs to the family of comets with intermediate dust-to-gas mass ratios. Dust release rates, determined by Newburn \& Spinrad (1989) on September 6, $1980(r=1.93 \mathrm{AU})$, and December 8, $1980(r=1.57$ $\mathrm{AU})$, are 47.84 and $226.1 \mathrm{~kg} / \mathrm{s}$ for parameters $\rho_{\mathrm{d}} \rho_{\mathrm{N}}=0.3$ $\mathrm{g}^{2} / \mathrm{cm}^{6}$ and $p(\lambda)=0.04$. The dust release rates determined for September 3.45, 1980, and December 10.35, 1980 , considering $\rho_{\mathrm{d}} \rho_{\mathrm{N}}=0.5 \mathrm{~g}^{2} / \mathrm{ch}^{6}$ and $p(\lambda)=0.04$, are about 60 and $222 \mathrm{~kg} / \mathrm{s}$ (Table 3 ) and compare well with those of Newburn \& Spinrad (1989).

Calculation of the mean effective dust particle size from $q_{\mathrm{d}}$ and $A_{\mathrm{d}} \cdot p(\lambda)$ indicates that the scattering at optical wavelengths in Comet Stephan-Oterma (1980 X) was due to dust particles with $a \approx 3.5 \mu \mathrm{m}$. This agrees with 

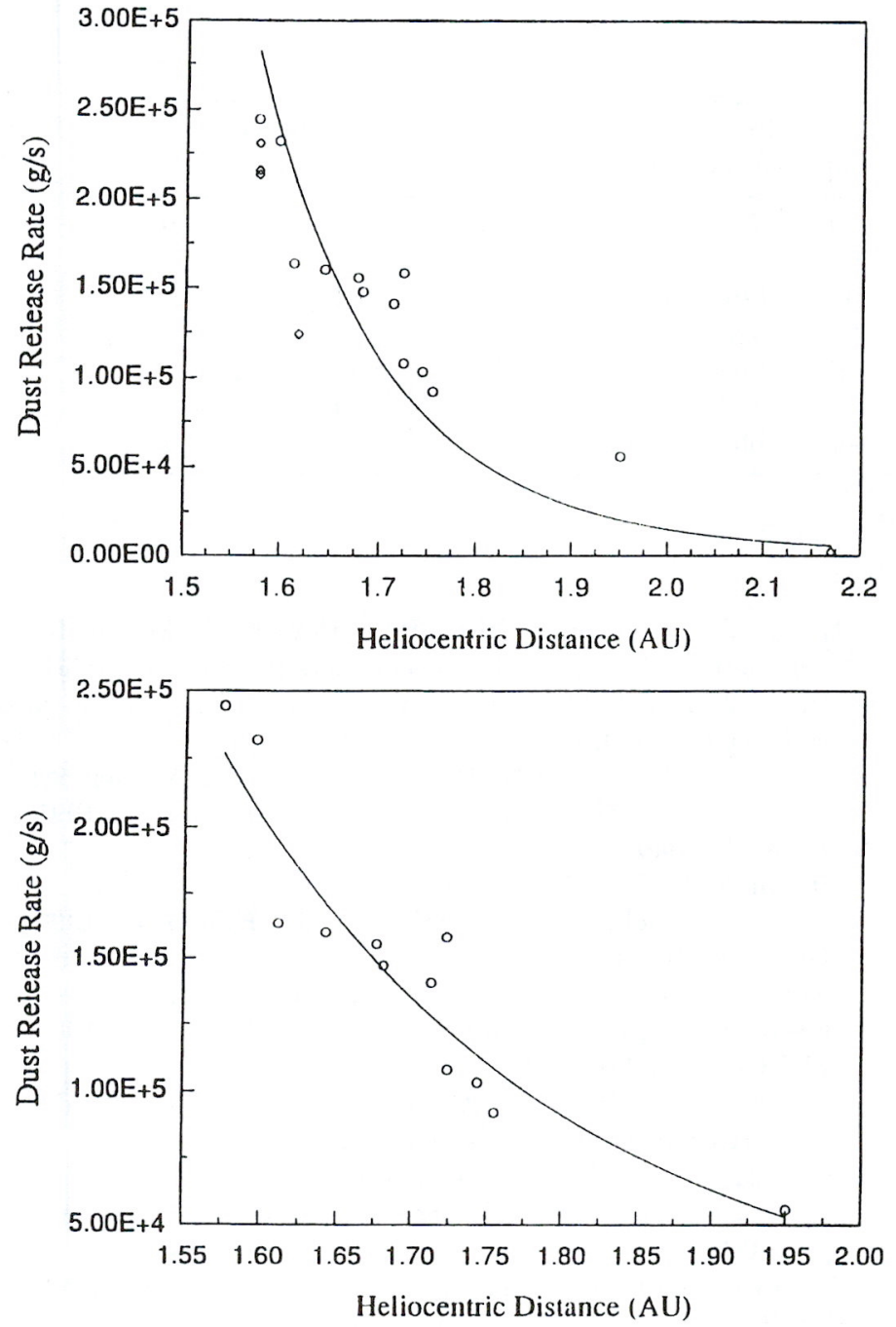

Fig. 6. Variation of dust release rate with heliocentric distance for Comet Stephan-Oterma (1980 X). a) Circles: Pre-perihelion, diamonds: Post-perihelion. b) Pre-perihelion only

the conclusion drawn from infrared studies by Veeder \& Hanner (1981).

The dust in Comet Stephan-Oterma (1980 X) was red relative to the Sun in the wavelength range $3300-5240 \AA$ for the whole observing period of Millis et al. (1982). On October 4.55 and $6.52,1980(r=1.755$ and $1.744 \mathrm{AU})$, the dust was blue relative to the Sun $\left(C E=-0.2^{\mathrm{mag}}\right.$ in the wavelength range $4120-5240 \AA$ ). Significant changes in dust color can also be noticed on October 12.53, 19.49, and $20.60,1980$, in the wavelength range $3675-4120 \AA$. The dust was blue relative to the Sun $\left(C E=-0.37^{\mathrm{mag}}\right)$ at these three dates and it remained slightly blue relative to the Sun after October 20.60, 1980, in the wavelength range $3675-4120 \AA$.

\section{Conclusions}

It is well known from observations that comets show large differences in the compositions of their comae and tails. Some comets have large plasma tails others have none, some are rich on dust others are dust-poor, etc. These differences have been ascribed to differences in the composition of their nuclei, i.e., they may have had a different place of origin or they may have formed at a different time. But since large areas of a comet nucleus appear to be inactive relative to other, smaller areas, the differences could also stem from inhomogeneities in the nucleus. Since our analyses indicate different mass ratios of dust-to-gas release rates as well as changes in the dust color for the same comet at different times, we interpret this behavior to be caused by inhomogeneities in each nucleus. For example, the collapse of regolith around an eroded active area may suddenly change the dust-to-gas ratio, the dust particle size distribution, and the composition of the dust.

In Table 3 we summarize the mean dust particle sizes based on Eq. (12) that account for the observed dust mass loss rates if all particles were of the same size. As mentioned in the discussions of the individual comets, these particle sizes are very similar to those reported by other observers. The mean particle size varies from comet to comet, but typically is between 1 and more than $10 \mu \mathrm{m}$. On the other hand, interstellar grains are much less than $1 \mu \mathrm{m}$ in size. Thus it appears that cometary dust particles may be aggregates of interstellar grains. Such aggregates may have formed in the solar nebula during the initial phases of planetesimal formation. Aggregation may have been enhanced also on the surface of comets as a result of cosmic and solar radiation by low-temperature sintering. In this process, the ice mantle on a grain tends to evaporate very slowly from positively curved (convex) surfaces and recondense on negatively curved (concave) surfaces where particles touch, thus forming an icy neck that holds the particles together in a very fluffy structure. Over long periods of time, organic materials may participate in the sintering process until a regolith layer forms on the nucleus making the surface heat-resistant and relatively inactive (i.e., the gas flux is small compared to that of an active region). Small dust particles, i.e., unprocessed (unsintered) dust may come from active areas during quiescent phases of the comet or from relatively inactive regions where the gas flux is too low to entrain large particles. Thus, small dust particles may be more closely related to the original dust as it was incorporated into a comet during its formation. Hence, quiescent phases may reveal more about the original composition of a comet nucleus, with an active area acting as a "window," letting us "view" the matter in the interior.

Since dust particles are the most likely condensation nuclei for the accumulation of ices, it is difficult to understand how a comet can be "dust free", i.e., have a very low ratio of dust-to-gas, $\chi$. It is thus interesting to 
Table 4. Correlations

\begin{tabular}{lcccccccc}
\hline Comet & $P^{*}$ & $\chi$ & $\begin{array}{c}a \\
{[\mu \mathrm{m}]}\end{array}$ & $\begin{array}{c}a_{\mathrm{m}} \\
{[\mathrm{cm}]}\end{array}$ & $\begin{array}{c}\text { Color } \\
\text { Excess }\end{array}$ & $\begin{array}{c}R_{\mathrm{N}} \\
{[\mathrm{km}]}\end{array}$ & $\begin{array}{c}\text { \% Active } \\
\text { Area }\end{array}$ & Comments \\
\hline Bowell (1982 I) & $\mathrm{L}$ & $0.3-2$ & $2-8$ & $1-14$ & red & 4 & 50 & blue when $\chi<0.4$ \\
Bradfield (1979 X) & 280 & $<0.1$ & $3-5$ & 28 & blue & 1 & 10 & Halley-type \\
Brorsen-Metcalf (1989 X) & $\mathrm{S}$ & $<0.1$ & $3-4$ & 9 & blue & 10 & 2 & \\
Giacobini-Zinner (1985 XIII) & $\mathrm{S}$ & $0.2-0.4$ & $3-5$ & 25 & red & 1.2 & 10 & pre-perihelion \\
Levy (1990 XX) & $\mathrm{L}$ & $0.6-1$ & $5.5-10$ & 16 & blue & 3 & 6 & post-perihelion \\
Levy (1990 XX) & $\mathrm{L}$ & $0.6-2$ & $5.5-12$ & 16 & red & 3 & 6 & 10 \\
Stephan-Oterma (1980 X) & $\mathrm{S}$ & $0.15-0.3$ & 3 & $4-6$ & blue & 5 & & \\
\hline
\end{tabular}

* Period: $\mathrm{S}=$ short-period $(<200 \mathrm{y}), \mathrm{L}=\operatorname{long}$-period $(>200 \mathrm{y})$.

speculate whether "dust free" comets have much more fine dust $(a<<1 \mu \mathrm{m})$ than the extrapolations of the size distributions from the detected dust in the visible and IR ranges of the usual observations suggest, making such comets richer on dust than suspected. If this is the case, the dust size distribution may be bimodal. In such a distribution the fine dust may be closely related to the interstellar dust, while the larger dust particles are typical cometary aggregates. The bimodal distribution may not be detectable in a dust-rich comet where it may be obliterated by the tail in the particle distribution.

Table 4 summarizes the results from this very small sample giving, for each comet, the orbital period, $P$, the mass range of dust-to-gas release rates, $\chi$, the mean particle size, $a$, responsible for the continuum flux, the maximum particle size $a_{\mathrm{m}}$, that can be entrained by coma gas escaping from the nucleus, and the color excess with respect to the Sun. The dust is blue relative to the Sun for comets with $\chi<0.1$, but can be blue for comets with $0.1<\chi<0.4$. The two long-period comets Bowell (1982 I) and Levy (1990 XX) are dustier than the other, short-period comets and the dust is red relative to the Sun except when they are far from the Sun and $\chi$ is small relative to the peak values.

Acknowledgements. P.D.S. is thankful to the Southwest Research Institute for its hospitality, to Dr. D.C. Boice for his help, to CNPq for financial support (Processo: 200020/92-7), and to the University of São Paulo for granting a leave of absence. G.C.S. is thankful to SUL for granting a leave of absence and to program PICD/CAPES/UEL for financial assistance. This work was supported by NASA grant NAGW-2370.

\section{References}

A'Hearn M.F., Cowan J.J., 1975, AJ 80, 852

A'Hearn M.F., Millis R.L., Birch P.V., 1981, AJ 86, 1559

A'Hearn M.F., Schleicher D.G., Feldman P.D., Millis R.L., Thompson D.T., 1984, AJ 89, 579

A'Hearn M.F., Hoban S., Birch P.V., Bowers C., Martin R., Klinglesmith D.A., 1986a, Nat 324, 649
A'Hearn M.F., Hoban S., Birch P.V., Bowers C., Martin R., Klinglesmith D.A., 1986b, Gaseous jets in comet P/Halley, Proc. 20th ESLAB Symposium on the Exploration of Halley's comet, ESA SP - 250, Vol. I., p. 483

Bailey M.E., Chambers J.E., Hahn G., 1992, MNRAS 254, 581

Baratta G.A., Catalano F.A., Leone F., Strazzulla G., 1991, A\&A 251, 349

Bobrovnikoff N.T., 1954, AJ 59, 357

Boice D.C., Huebner W.F., Sablik M.J., Konno I., 1990, Geophys. Res. Lett. 17, 1813

Cowan J.J., A'Hearn M.F., 1979, Moon Planets 21, 155

de Freitas Pacheco J.A., Landaberry S.J.C., Singh P.D., 1988, MNRAS 235, 457

Delsemme A.H., 1982, in: Comets, Wilkening L.L. (ed.). University of Arizona Press Tucson, AZ., p. 85

DiSanti M.A., Fink U., 1991, Icarus 91, 105

Eberhardt P., Krankowsky D., Schulte W., et al., 1987, A\&A 187,481

Fulle M., Pasian F., Benvenuti P., 1992, Ann. Geophys. 10, 145

Gehrz R.D., Ney E.P., 1992, Icarus 100, 162

Geiss J., 1988, Rev. Mod. Astron. 1, 1

Hanner M.S., Veeder G.J., Tokunaga A.T., 1992, AJ 104, 386

Huebner W.F., Keady J.J., Lyon S.P., 1992, Solar Photo Rates for Planetary Atmospheres and Atmospheric Pollutants. Kluwer Academic Publishers

Huebner W.F., Keller H.U., Jewitt D.C., Klinger J., West R., 1993. The Activity of Distant Comets, Southwest Research Institute report

Jessberger E.K., Kissel J., Rahe J., 1989, in: Origin and Evolution of Planetary and Satellite Atmospheres, Atreya S.K., Pollack J.B., and Mathews M.S. (eds.). The University of Arizona Press, Tucson, AZ, p. 167

Jewitt D.C., Soifer B.T., Neugebauer G., Mathews K., Danielson G.E., 1982, ApJ 87, 1854

Jewitt D.C., 1984, Icarus 60, 373

Jewitt D.C., Luu J., 1990, ApJ 365, 738

Keller H.U., Arpigny C., Barbieri C., et al., 1986, Nat 321, 320

Landaberry S.J.C., Singh P.D., de Freitas Pacheco J.A., 1991, A\&A 246, 597

Lynch D.K., Russell R.W., Hackwell J.A., Hanner M.S., Hammel H.B., 1992, Icarus 100, 197

Lynch D.K., Hanner M.S., Russell R.W., 1992, Icarus 97, 269 Marsden B.G., 1990, IAU Circular 5085 
McFadden L.A., A'Hearn M.F., Feldman P.D., et al., 1987, Icarus 69,329

Meech K.J., Jewitt D.C., 1987, Nat 328, 506

Meier R., Krankowsky D., Eberhardt P., Schulte W., Lämmerzahl P., Hodges R.R., 1993, A\&A 277, 677

Millis R.L., A'Hearn M.F., Thompson D.T., 1982, AJ 87, 1310

Morris C.S., 1980, Internat. Comet Quart. 2, 24

Mukai T., 1986, A\&A 164, 397

Newburn Jr. R.L., Spinrad H., 1985, AJ 90, 2591

Newburn Jr. R.L., Spinrad H., 1989, AJ 97, 552

Roettger E.E., Feldman P.D., A'Hearn M.F., Festou M.C., McFadden L.A., Gilmozzi R., 1989, Icarus 80, 303

Schleicher D.G., Millis R.L., Birch P.V., 1987, A\&A 187, 531

Schleicher D.G., Millis R.L., Osip D.J., Birch P.V., 1991, Icarus 94,511
Singh P.D., de Almeida A.A., Huebner W.F., 1992, AJ 104, 848

Singh P.D., Huebner W.F., Costa R.D.D., Landaberry S.J.C., de Freitas Pacheco J.A., 1996, Plan. Space Sci. (in press)

Snyder L.E., Palmer E., de Pater I., 1989, AJ 97, 246

Vaisberg O., Smirnov V., Omelchenko A., 1986, Spatial distribution of low-mass dust particles $\left(m<10^{-10} \mathrm{gm}\right)$ in Comet Halley coma. Proc. 20th ESLAB Symposium on the Exploration of Halley's Comet. ESA SP - 250, Vol. II, p. 17 Veeder G.J., Hanner M.S., 1981, Icarus 47, 381

Yabushita S., Wada K., 1987, Earth Moon Planets 40, 303

Weaver H.A., A'Hearn M.F., Feldman P.D., et al., 1992, Icarus 97,85

Whipple F.L., 1978, Moon Planets 18, 343

Table 1. Basic observational data.

Column 1: Data set.

Column 2: Date of observation (UT).

Column 3: Heliocentric distance, $r$ (AU).

Column 4: Geocentric distance, $\Delta$ (AU).

Column 5: Phase angle (o).

Column 6: Projected diaphragm radius, $s(\mathrm{~km})$.

Column 7: Dimensionless parameter for particle size distribution (see: de Freitas Pacheco, et al., 1988, MNRAS 235, 547).

Column 8: Dimensionless phase function (see: de Freitas Pacheco, et al., 1988, MNRAS 235, 547).

Table 2. Area - geometric albedo product.

Column 1: Data set.

Column 2: Measured continuum flux $\left(\operatorname{erg~} \mathrm{cm}^{-2} \mathrm{~s}^{-1} \hat{\mathrm{A}}^{-1}\right)$.

Column 3: Dimensionless phase function of the nucleus, $\phi_{\mathrm{N}}(\theta)$.

Column 4: Nucleus contribution to area - albedo product $\left(\mathrm{cm}^{2}\right)$.

Column 5: Total area - albedo product $\left(\mathrm{cm}^{2}\right)$ from dust coma and nucleus.

Column 6: Area - albedo product $\left(\mathrm{cm}^{2}\right)$ for dust coma alone.

Table 3. Dust mass release rates.

Column 1: Data set.

Column 2: Gas release rate $(\mathrm{g} / \mathrm{s})$.

Column 3: Maximum size of dust particles, $a_{\mathrm{m}}(\mathrm{cm})$.

Column 4: Equivalent dust particle size, $a(\mu \mathrm{m})$, to give the same dust release rate as the actual particle size distribution.

Column 5: Dust release rate $(\mathrm{g} / \mathrm{s})$.

Column 6: Dust-to-gas mass ratio (dimensionless). 